GSA Data Repository 2016065

\title{
Universal scaling of fluid permeability during volcanic welding and sediment diagenesis
}

Fabian B. Wadsworth et al.

\section{PARAMETERS USED IN EQUATION 2 (Main Text)}

In the main text, Equation 2 requires knowledge of the functional form of $y_{0}, y_{1}, y_{2}$ and $y_{3}$, all of which depend on $\phi$ only as follows (Torquato, 2013)

$$
\begin{gathered}
y_{0}=\frac{2-\phi+(1-\phi)^{2}-(1-\phi)^{3}}{\phi^{3}}, \\
y_{1}=\frac{(1-\phi)\left(3(1-\phi)^{2}+4 \phi-7\right)}{2 \phi^{3}}, \\
y_{2}=\frac{(1-\phi)^{2}(1+\phi)}{2 \phi^{3}}, \\
y_{3}=-\left(y_{0}+3 y_{1}+12 y_{2}\right) .
\end{gathered}
$$

S.Eq. 1

\section{SOLUTION FOR $a$ WHEN $R$ IS POLYDISPERSE}

Here we acknowledge the fact that for many cases of natural import, the particle size range deposited in beds of densifying volcanic ash or sediments is not monodisperse. To do this, we again use the statistical model for random heterogeneous media (Torquato, 2013) to adapt Eq. 2 (main text) as follows

$$
F(x)=\exp \left[2 \vartheta(\phi-1)\left(z_{0} \beta^{3}+z_{1} \beta^{2}+z_{2} \beta\right)\right],
$$

and now, in contrast with the main text, $\beta=(1+x) / 2$ and $\vartheta=\left\langle R^{2}\right\rangle\langle R\rangle /\left\langle R^{3}\right\rangle$, where $\left\langle R^{n}\right\rangle$ is the $n^{\text {th }}$ moments of the distribution of $R$ (the mean radius being $n=1$, i.e. $\langle R\rangle$ ). As with Eq. 2 in the main text, S.Eq. 2 uses coefficients that depend on $\phi, z_{0}, z_{1}$, and $z_{2}$ which are defined as follows

$$
\begin{gathered}
z_{0}=\frac{4 \phi\langle R\rangle^{2}(\phi+3 \vartheta(1-\phi)) /\left\langle R^{2}\right\rangle+8(\vartheta(1-\phi))^{2}}{\phi^{3}}, \\
z_{1}=\frac{6 \phi\langle R\rangle^{2} /\left\langle R^{2}\right\rangle+9 \vartheta(1-\phi)}{\phi^{2}}, \\
z_{2}=\frac{3}{\phi} .
\end{gathered}
$$

As in the main text, the pore-size can be found from $\left\langle x^{n}\right\rangle=n \int_{0}^{\infty} x^{n-1} F(x) d x$ such that the characteristic pore size (the mean) for polydisperse particle beds is $a=R\langle x\rangle$. 


\section{ALTERNATIVE SCALING ARGUMENTS}

Here, for completeness, we demonstrate that our scaling presented in the main text is the most appropriate. To do this we show variations on our scaling concept and explain each one in turn. In the main text we present the result when we use the initial value of $R$ to compute $a$ at the initial value of $\phi$. And in doing so we state that $R$ is for impenetrable hard spherical particles and to compute the specific surface area, we state that $a$ is for fully penetrable spherical pores. The symbols in all figures are as they appear in the main text to which the reader is referred for references.

\section{i. Data repository alternative method (1) - overlapping particles}

If we disregard the pores, and thus neglect to compute $a$, then we can compute the surface area from the particles assuming they are overlapping spheres with radius $R$. To do this, Eq. 3 in the main text is replaced by

$$
s(R)=-\frac{3 \phi \ln \phi}{R}
$$

In doing so we find the scaling result in Supplementary Figure DR1. This clearly is ineffective, not only because it disagrees with the scaling proposed by Martys et al. (1994) but because it does not provide universal collapse of the data.

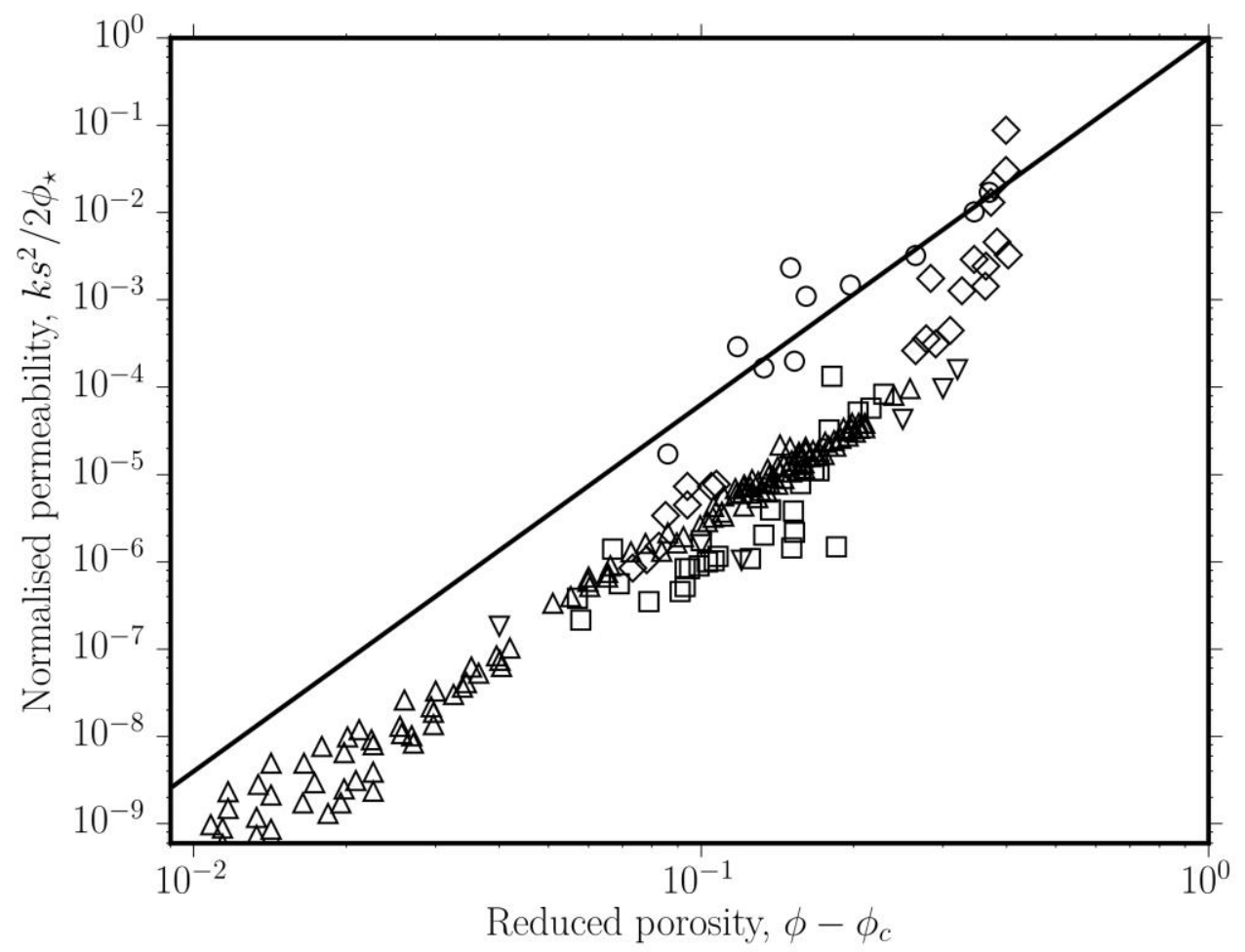




\section{ii. Data repository alternative method (2) - non-overlapping particles; non-overlapping pores}

If we return to the method explicated in the main text, for which $R$ is used to compute the pore radius $a$ assuming the particles are non-overlapping spheres at an initial $\phi$ and, in the computation of the specific surface area, we assume that the pores are non-overlapping spheres (note: in the main text we revise this assumption and consider that the pores are overlapping spheres) then we find the scaling result in Supplementary Figure DR2. This is close to the result presented in the main text but here the goodness of agreement is slightly worse across all data.

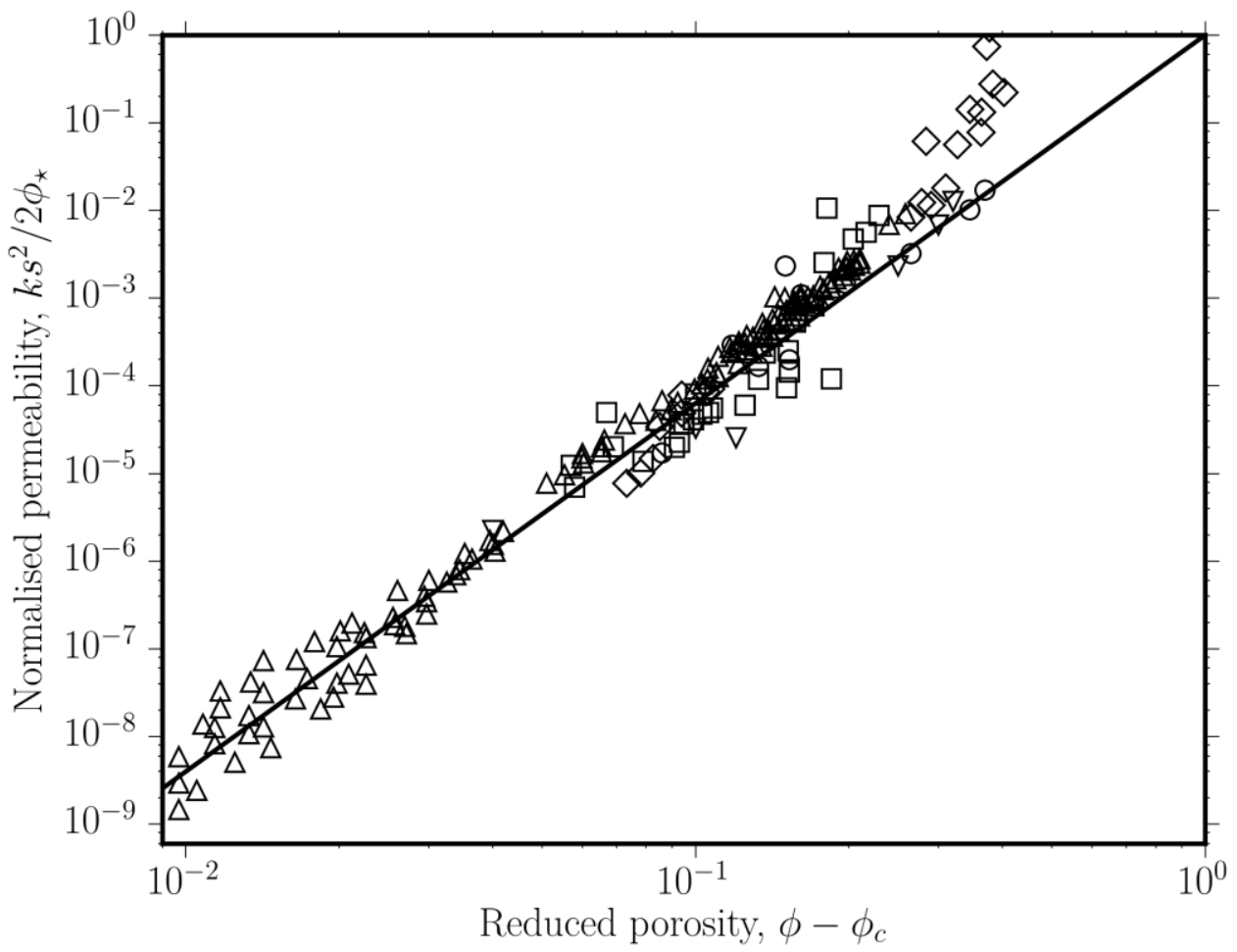

Supplementary Figure DR2

iii. Data repository alternative method (3) - evolving pore size between overlapping particles for overlapping or non-overlapping pores

Now if instead of taking a constant pore size $a$ computed from the particle sizes $R$ at the initial value of $\phi$, we rather compute the pore size continuously as a function of $\phi$, we can compute a $s$ directly assuming that the particles are overlapping. To do this, we replace Eq. 2 from the main text with 


$$
F(x)=\frac{\exp \left(\beta^{3} \ln (1-\phi)\right)}{\phi}
$$

and we use Eq. 3 from the main text to compute $s$.

This yields a pore size $a$ that is evolving for each data point. If we then assume that the pores are overlapping or non-overlapping, respectively, we arrive at the scaling results in Supplementary Figures DR3a and DR3b, respectively. As before, these assumptions provide a data scatter with lower agreement with the solution of Martys et al., (1994).
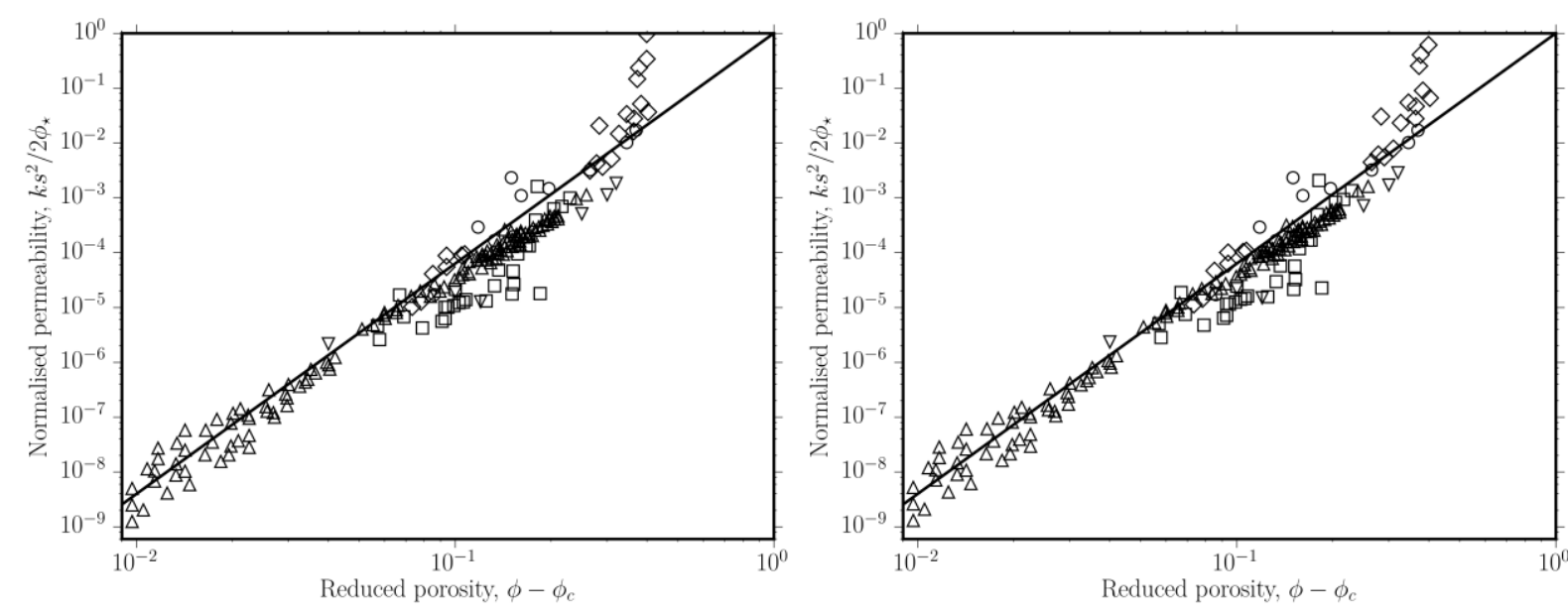

Supplementary Figure DR3a and DR3b

\section{iv. Data repository alternative method (4) - the Stokes permeability for dispersions}

Finally, if we disregard the scaling discussed in the main text and instead attempt to normalize the permeability using the Stokes dilute limit permeability $k_{s}=2 R^{2} /[9(1-\phi)]$ for granular media, then we arrive at Supplementary Figure DR4. As with other scaling attempts discussed here, this works less well than the one provided in the main text. Here for completeness the proposal of Martys et al. (1994) that $k=k_{s}\left(\phi-\phi_{c}\right)^{4}$ is provided to guide the eye. 


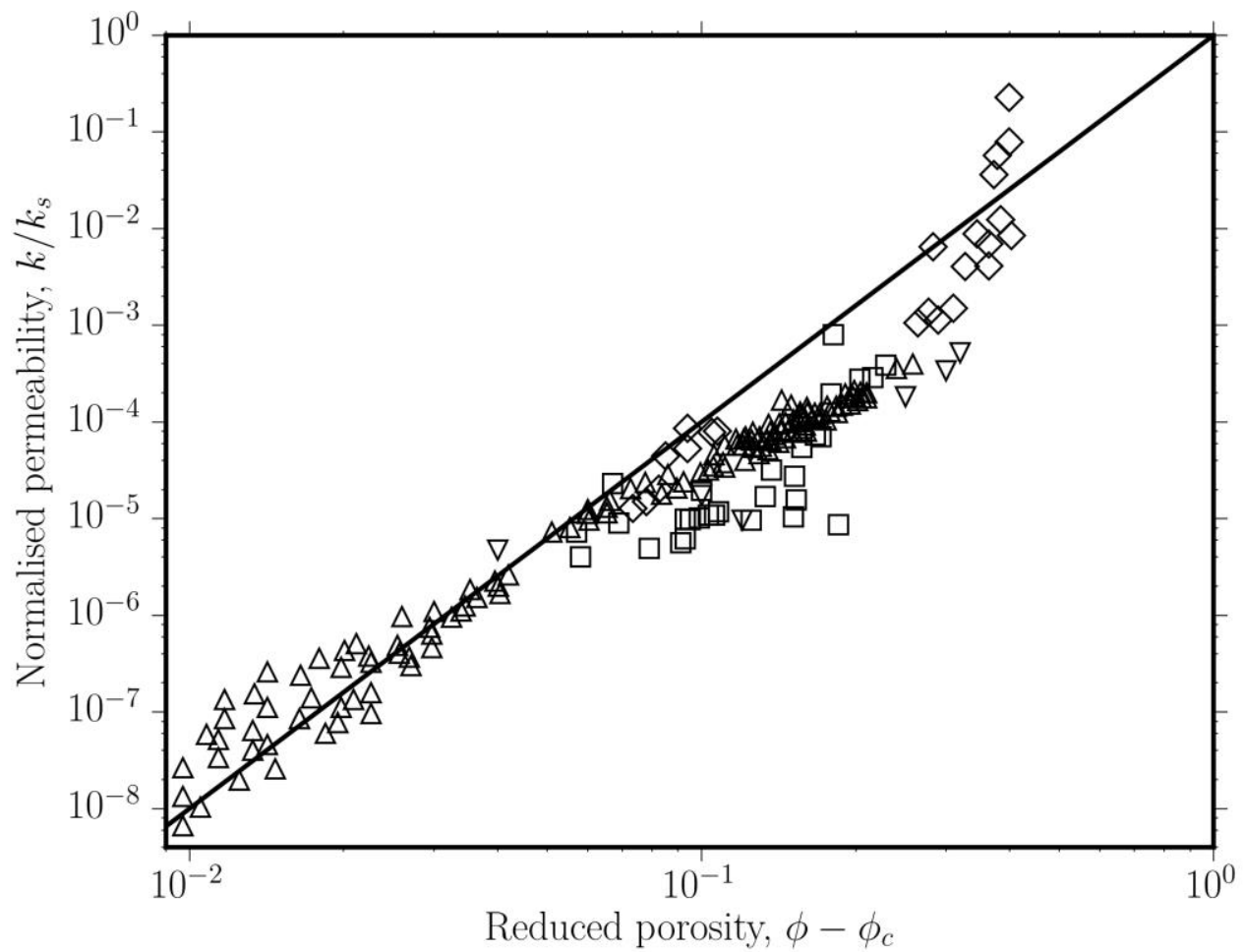

Supplementary Figure DR4 


\section{WHICH MODEL IS BEST?}

Here we quote the coefficient of determination $r^{2}$ for each normalization method described in the main text and in the data repository to show that the method chosen is the most effective $\left(r^{2}=0.95\right)$.

\begin{tabular}{|l|l|}
\hline Supplementary Table & \\
\hline Normalisation method & Coefficient of determination, $r^{2}$ \\
\hline As described in main text (Inset; Figure 3) & 0.85 \\
\hline As described in main text (Figure 3) & $\mathbf{0 . 9 6}$ \\
\hline Data repository method (1) & 0.56 \\
\hline Data repository method (2) & 0.94 \\
\hline Data repository method (3a) & 0.92 \\
\hline Data repository method (3b) & 0.93 \\
\hline Data repository method (4) & 0.75 \\
\hline
\end{tabular}

\section{REFERENCES CITED IN THE DATA REPOSITORY}

Torquato, S., 2013, Random heterogeneous materials: microstructure and macroscopic properties, Springer Science \& Business Media. 